\title{
On the Pricing Formula for the Perpetual American Volatility Option Under the Mean-reverting Processes
}

\author{
Hsuan-Ku Liu, Tse-Yu Lin and Yen-Lung Tsai*
}

\begin{abstract}
This paper studies the properties of the parabolic free-boundary problem arising from pricing of American volatility options in mean-reverting volatility processes. When the volatility index follows the mean-reverting square root process (MRSRP), we derive a closed-form pricing formula for the perpetual American power volatility option. Moreover, an artificial neural network (ANN) approach is extended to find an approximate solution of the free boundary problem arising from pricing the perpetual American option. The comparison results demonstrates that the ANN provides an accurate approach to approximate solution for the free boundary problem.
\end{abstract}

\section{Introduction}

1.1. The probability density function and expectation

In the case of mean-reverting square root process (MRSRP), the index process under the Martingale measure $Q$ is presented as

$$
d x_{t}=\beta\left(m-x_{t}\right) d t+\sigma \sqrt{x_{t}} d w_{t}
$$

with $\beta, m$ and $\sigma$ representing the speed of mean-reversion, the long-run mean, and the volatility of the volatility index, respectively [3,6]. Here, $x$ denotes the index of volatility, $t$ denotes the time-to-maturity and $d w_{t}$ denotes an increment in the Wiener process under the Martingale probability measure $Q$. The probability density function of $x$ at the future time $T$ under the current time $t$ is given as (see Cox et al. [1])

$$
p\left(x_{T}, x_{t} \mid \beta, m, \sigma\right)=c e^{-u-v}\left(\frac{v}{u}\right)^{q / 2} I_{q}(2 \sqrt{u v})
$$

where $c=\frac{2 \beta}{\sigma^{2}\left(1-e^{-\beta(T-t)}\right)}, u=c x_{t} e^{-\beta(T-t)}, v=c x_{T}, q=\frac{2 \beta m}{\sigma^{2}}-1$ and $I_{q}$ is the modified Bessel function of the first kind of order $q$.

Received December 24, 2019; Accepted August 12, 2020.

Communicated by Jenn-Nan Wang.

2010 Mathematics Subject Classification. 60H10, 60H15.

Key words and phrases. free boundary problem, American volatility options, neural network approach. This work was partially supported by the MOST under Grant No. 107-2115-M-152-001.

*Corresponding author. 
In the case of mean-reverting $3 / 2$ volatility process, the index process under the Martingale measure $Q$ is given as follows:

$$
d x_{t}=\left(\alpha x_{t}-\beta x_{t}^{2}\right) d t+k x_{t}^{3 / 2} d w_{t},
$$

where $\alpha>0, \beta>0$ and $k \neq 0$ are constants. This model has a nonlinear drift so that it exhibits substantial nonlinear mean-reverting behavior when the volatility is above its long-run mean. Hence, after a large volatility spike, the volatility can potentially quickly decrease, while after a low volatility period it can be slow to increase. Applying change of variables $y_{t}=1 / x_{t}, y$ follows the following MRSRP

$$
d y_{t}=\left(\left(k^{2}+\beta\right)-\alpha y_{t}\right) d t-k \sqrt{y_{t}} d w_{t} .
$$

Based on (1.1), the probability density function of $y$ is then presented as follows:

$$
p\left(y_{T}, y_{t} \mid \alpha, \frac{k^{2}+\beta}{\alpha},-k\right)=c e^{-u-z}\left(\frac{z}{u}\right)^{q / 2} I_{q}(2 \sqrt{u z}),
$$

where $c=\frac{2 \alpha}{k^{2}\left(1-e^{-\alpha(T-t)}\right)}, u=c y_{t} e^{-\alpha(T-t)}, z=c y_{T}$ and $q=1+\frac{2 \beta}{k^{2}}$ (see Goard and Mazur [5]). Since the probability density function of the MRSRP and the mean-reverting $3 / 2$ processes are given in 1.1 and $(1.2)$, respectively, the value of a European option can be obtained as

$$
V(x, t)=e^{-r(T-t)} \mathbb{E}^{Q}\left[\psi\left(x_{T}\right) \mid x_{t}=x\right],
$$

where $\psi(x)$ is the payoff function of the European volatility option and $\mathbb{E}^{Q}$ denotes the expectation under the martingale measure $Q$.

\subsection{The solution of partial differential equations}

Except considering the probability density function to find the expectation for the price, the pricing formula of the European volatility option is also the solution of partial differential equations. When the volatility index follows the MRSRP, the pricing equation of the volatility option $V(x, t)$ is presented as

$$
\left(\mathcal{L}_{0}^{M}-\frac{\partial}{\partial t}\right) V=0, \quad 0 \leq x<\infty, 0<t<\infty,
$$

where $\mathcal{L}_{0}^{M}$ is defined as

$$
\mathcal{L}_{0}^{M} \equiv \frac{1}{2} \sigma^{2} x \frac{\partial^{2}}{\partial x^{2}}+\beta(m-x) \frac{\partial}{\partial x}-r .
$$

The fundamental solution of (1.3) is given by Feller [4]. When the volatility index follows the mean-reverting $3 / 2$ volatility model, the value $V(x, t)$ of the volatility option can also be obtained by solving the following parabolic equation

$$
\left(\mathcal{L}_{0}^{Q}-\frac{\partial}{\partial t}\right) V=0, \quad 0 \leq x<\infty, 0<t<\infty
$$


where the operator $\mathcal{L}_{0}$ is given in the form

$$
\mathcal{L}_{0}^{Q} \equiv \frac{1}{2} k^{2} x^{3} \frac{\partial^{2}}{\partial x^{2}}+\left(\alpha x-\beta x^{2}\right) \frac{\partial}{\partial x}-r .
$$

The coefficients are all continuously differentiable and $\frac{1}{2} k^{2} x^{3}>0$ for $0<x<\infty, k \neq 0$ and $r>0$. By setting $x=1 / y, \mathcal{L}_{0}^{Q} V(x)$ can be changed to $\mathcal{L}_{0}^{M} V(y)$.

The closed-form expression for the value of a European volatility call option was proposed by Grunbichler and Longstaff [6], who found that the price of a volatility call option can be below its intrinsic value and that the traditional put-call parity relation does not hold for these options. This is because the volatility is not the price of a traded asset. However, the value of the American style volatility call option, unlike the European option, is bounded below by its early exercise payoff. Evidently, the lower bound is a consequence of the possibility of immediate exercise. Moreover, the European option still has value as the volatility decreases to zero in the MRSRP case. Detemple and Osakwe 3] said that the reason for this difference is the multiplicative impact of the uncertainty of future volatility. They also showed that the price of the American style volatility call $(\psi(x)=\max \{x-K, 0\})$ is an increasing function of the time-to-maturity.

\subsection{The free boundary problem for pricing an American volatility option}

For the American-style option, an entirely satisfactory analytic solution has not been found for the MRSRP model and the mean-reverting 3/2-volatility model, even though several researchers have concentrated on finding the properties of the value as well as the early exercise boundary for American options. Liu [10] proposed the properties of the price and the early exercise boundary for the American volatility put option $(\psi(x)=\max \{K-x, 0\})$ when the volatility index satisfies the mean-reverting $3 / 2$ volatility process.

In this paper, we consider the pricing problem for the American volatility call with the payoff function $\psi(x)=\max \left\{x^{n}-K, 0\right\}, n \in \mathbb{Z}$. Applying $\mathcal{L}_{0}^{M}$ to $\psi(x)$ yields that

$$
\mathcal{L}_{0}^{M} \psi(x)= \begin{cases}-(\beta n+r) x^{n}+\left(\frac{1}{2} \sigma^{2}(n-1)+\beta m\right) n x^{n-1}+r K & \text { if } x^{n}>K, \\ 0 & \text { if } x^{n}<K .\end{cases}
$$

Since $r K>0$ and $\mathcal{L}_{0}^{M} \psi(\xi) \rightarrow-\infty$ as $\xi \rightarrow \infty$ if $\beta n+r>0$. This implies that there exists $d>0$ such that

$$
\mathcal{L}_{0}^{M} \psi(x) \begin{cases}>0 & \text { for } 0<x<d \\ <0 & \text { for } d<x<\infty\end{cases}
$$

Precisely, this paper examines the following one-dimensional free boundary problem for linear parabolic equations arising from the problem of valuing an American-style volatility option in the models of MRSRP. Define $\mathcal{L}=\mathcal{L}_{0}^{M}-\frac{\partial}{\partial t}$. 
Let $u(x, t)$ and $s(t)$ be the price and the early exercise price of the American volatility power call. For the case of $n>0$, we consider the following free boundary problem

$$
\begin{cases}\mathcal{L} u=0, & 0<x<s(t), 0<t<\infty \\ u(x, t)>x^{n}-K, & 0<x<s(t), 0<t<\infty \\ u(x, 0)=x^{n}-K, & 0 \leq x \leq s(0) \\ u(s(t), t)=s^{n}(t)-K, & 0 \leq t<\infty \\ \frac{\partial u}{\partial x}(s(t), t)=\psi^{\prime}(s(t)), & 0 \leq t<\infty\end{cases}
$$

Since $u(x, t)$ denotes the price of an American volatility power call, the condition $u(0, t)<\infty$ is added in the model. These additional condition will be used in finding the pricing formula of the corresponding perpetual American option.

For the case of $n<0$, we have $x^{n}>K$ if $x<\frac{1}{\sqrt[n]{K}}$. Hence, the value of the American volatility option satisfies the following free boundary problem

$$
\begin{cases}\mathcal{L} u=0, & s(t)<x<\infty, 0<t<\infty, \\ u(x, t)>x^{n}-K, & s(t)<x<\infty, 0<t<\infty, \\ u(x, 0)=x^{n}-K, & s(0) \leq x \leq \infty \\ u(s(t), t)=s^{n}(t)-K, & 0 \leq t<\infty \\ \frac{\partial u}{\partial x}(s(t), t)=\psi^{\prime}(s(t)), & 0 \leq t<\infty\end{cases}
$$

In the mean reverting $3 / 2$ volatility process, the pricing problem for the American volatility option can be considered by changing the variable $x=1 / y$. It would be interesting to consider the properties of the value as well as the early exercise boundary of American volatility power options while the properties have not been mentioned in the case of the MRSRP and the mean-reverting $3 / 2$ volatility process.

In the cases of the MRSRP and the mean-reverting $3 / 2$ volatility process, we derive a closed-pricing formula for the perpetual American volatility power option, where the early exercise price can be obtained iteratively. Moreover, we consider neural network (NN) approach to the solution of the free boundary differential equation arising from pricing a perpetual American volatility option under the MRSRP. The numerical results show that the ANN approach is an accurate approach for pricing the American volatility option in the case of MRSRP. This NN approach can also be applied to approximate the pricing formula of the perpetual American option under the different process. In future studies, our results can be applied to consider the properties of other American-style derivatives with the payoff function satisfying (1.5) in the cases of the MRSRP and the mean-reverting $3 / 2$ volatility process. 


\section{Properties of the solution}

Let $\mathcal{T}$ denote the set of all stopping time $\tau$ for the process. The value of an American-style option is obtained by evaluating the following optimization problem

$$
\widehat{u}(x, t)=\sup _{\tau \in \mathcal{T}} \mathbb{E}^{Q}\left[\psi\left(x_{\tau}\right) \mid x(t)=x\right] .
$$

Kotlow [9] showed that the solution $(s, u)$ to Problem 1.6 resolves the optimization problem 2.1) by setting

$$
\widehat{u}(x, t)= \begin{cases}u(x, t) & \text { if }(x, t) \in C \\ \psi(x) & \text { if }(x, t) \in \bar{Q}-C,\end{cases}
$$

where $\bar{Q}=(0, \infty) \times(0, \infty)$ and $C=\{(x, t) \mid 0<x<s(t), 0<t<\infty\}$.

Let $\{s, u\}$ be the solution to Problem 1.6 and denote $C$, namely the continuation region, as

$$
C=\{(x, t) \mid 0<x<s(t), 0<t<\infty\} .
$$

Applying results of Kotlow 9] directly to (1.6), we obtained the following theorems.

Theorem 2.1. Let $\{s, u\}$ be a solution of (1.6). They have the following properties:

(a) $u_{t}>0$ in $C$.

(b) $s(0)=d$ and $s(t)>d$ for $0<t<\infty$.

(c) $s(t)$ is a non-decreasing function.

(d) There exists an $s^{\infty} \in(d, \infty)$ such that $s(t) \rightarrow s^{\infty}$ uniformly as $t \rightarrow \infty$ if $\lim \sup _{\xi \rightarrow \infty}\left[\mathcal{L}_{0}^{M} \psi(\xi)\right]<0$ and $\beta n+r>0$.

In the case of the American put option, Liu [10] provided the properties for the price as well as the early exercise boundary under the mean-reverting $3 / 2$ volatility model. When the payoff function satisfies (1.5), we obtained the following theorem by modifying the proof of Theorem 2.3 in [10]. The following theorem includes the call option and the power call option in the MRSRP or the mean-reverting $3 / 2$ volatility models.

Theorem 2.2. Let $\{s, u\}$ be a solution of $(1.6)$. Then

(a) $s(t)$ is a strictly increasing function.

(b) $u_{x}(x, t)>0$ for $(x, t) \in C$.

(c) When $\beta>0, u_{x}(x, t)<\psi^{\prime}(x)$ for $(x, t) \in C_{d}$, where $C_{d}=\{(x, t) \in C \mid x>d\}$. 
According to Theorems 2.1 and 2.2, we propose properties for the value and the early exercise boundary of an American volatility power option in the MRSRP (Theorem 2.3) and the mean-reverting $3 / 2$ volatility process (Theorem 2.5). The similar results for the American volatility call can also be founded in Detemple and Kitapbayev [2].

Theorem 2.3. Let $u(x, t)$ and $s(t)$ be the value and the early exercise boundary of an American volatility power option in the MRSRP. When $\beta n+r>0$, we have the following properties.

(a) The value $u(x, t)$ increases with an increase in both the time-to-maturity.

(b) The value $u(x, t)$ increases (decreases, respectively) with an increase in the volatility index $x$ for $n>0$ (for $n<0$, respectively).

(c) The early exercise boundary s(t) strictly increases (decreases, respectively) with an increase in the time-to-maturity for $n>0$ (for $n<0$, respectively).

(d) The early exercise boundary $s(t)$ is bounded by $d$ and $s^{\infty}$, where $s^{\infty}$ is the exercise boundary of its corresponding perpetual American option.

(e) The early exercise boundary starts at $d$, that is $s(0)=d$.

Proof. The coefficients of 1.3 are all continuously differentiable and $\frac{1}{2} \sigma^{2} x>0$ for $0<$ $x<\infty$ and $r>0$. To show that the value and the early exercise boundary of an American volatility power option satisfy Theorems 2.1 and 2.2 , it suffices to show that there exists a $d$ in $\mathbb{R}$ such that $\mathcal{L}_{0}^{M} \psi(x)$ satisfies

$$
\mathcal{L}_{0}^{M} \psi(x) \begin{cases}>0 & \text { for } 0<x<d \\ <0 & \text { for } d<x<\infty\end{cases}
$$

for some $d>0$.

Applying $\mathcal{L}_{0}^{M}$ to $\psi(x)=\max \left\{x^{n}-K, 0\right\}$ for a volatility power option yields that

$$
\mathcal{L}_{0}^{M} \psi(x)= \begin{cases}-(\beta n+r) x^{n}+\left(\frac{1}{2} \sigma^{2}(n-1)+\beta m\right) n x^{n-1}+r K & \text { if } x^{n}>K, \\ 0 & \text { if } x^{n}<K .\end{cases}
$$

Let $f(x)=-(\beta n+r) x^{n}+\left(\frac{1}{2} \sigma^{2}(n-1)+\beta m\right) n x^{n-1}+r K$. We have $f(0)=r K>0$ and $\lim _{x \rightarrow \infty} f(x)=-\infty$ since $-(\beta n+r)<0$. Since $f$ is a continuous function on $\mathbb{R}, f$ has at least one positive root. Moreover, we have $f^{\prime}(x)=x^{n-2}\left[-n(\beta n+r) x+\left(\frac{1}{2} \sigma^{2}(n-\right.\right.$ $1)+\beta m) n(n-1)]$. This implies that $f(x)$ increases as $x<\frac{\left(\frac{1}{2} \sigma^{2}(n-1)+\beta m\right)(n-1)}{\beta n+r}$ and decreases as $x>\frac{\left(\frac{1}{2} \sigma^{2}(n-1)+\beta m\right)(n-1)}{\beta n+r}$. By the continuity of $f$, we obtained that $f$ has exactly one positive root, say $d^{\prime}$. Then we can define $d=\max \left\{K^{1 / n}, d^{\prime}\right\}$ for $n>0$ and $d=\min \left\{K^{1 / n}, d^{\prime}\right\}$ for $n<0$. 
Remark 2.4. For the American volatility call option with $\psi(x)=\max \{x-K, d\}$, we have $d=\max \left\{K, \frac{r}{r+\beta} K\right\}=K$ in the case of the MRSRP. This is because $\beta>0$ and $\mathcal{L}_{0}^{M} \psi(x)=-(\beta+r) x+r K$ for $x>K$.

Now, we consider properties of an American volatility power option in the meanreverting $3 / 2$ volatility process.

Theorem 2.5. When $\frac{1}{2} k^{2}(n-1)<\beta$ and $u>0$, the value $u(x, t)$ and the early exercise boundary $s(t)$ of an American power option have the same properties of (a) to (d) in Theorem 2.3 with the volatility following the mean-reverting $3 / 2$ volatility process.

Proof. Applying $\mathcal{L}_{0}^{Q}$ to $\psi(x)=\max \left\{x^{n}-K, 0\right\}$ for a volatility power option yields that

$$
\mathcal{L}_{0}^{Q} \psi(x)= \begin{cases}\left(\frac{1}{2} k^{2} n(n-1)-\beta n\right) x^{n+1}+(\alpha n-r) n x^{n}+r K & \text { if } x>K \\ 0 & \text { if } x<K\end{cases}
$$

Let $f(x)=n A x^{n+1}+(\alpha n-r) n x^{n}+r K$, where $A=\frac{1}{2} k^{2}(n-1)-\beta$. Then $f^{\prime}(x)=n x^{n}[(n+$ 1) $A x+(\alpha n-r)]$ and $f(0)=r K>0$. Since $A<0$, we have $\lim _{x \rightarrow \infty} f(x)=-\infty$ and $f^{\prime}(x)>0$ if $x<\frac{r-\alpha n}{(n+1) A}$ and $f^{\prime}(x)<0$ if $x>\frac{r-\alpha n}{(n+1) A}$. Hence $f(x)$ increases with $x<\frac{r-\alpha n}{(n+1) A}$ and deceases with $x>\frac{r-\alpha n}{(n+1) A}$. Therefore, we obtained that $f(x)$ has exactly one positive root, say $d^{\prime}$. Then we can define $d=\max \left\{K^{1 / n}, d^{\prime}\right\}$ for $n>0$ and $d=\min \left\{K^{1 / n}, d^{\prime}\right\}$ for $n<0$.

Remark 2.6. According to Theorem 2.5, the value of $s(0)=d$ for the American call option with $\psi(x)=\max \{x-K, 0\}$ is obtained as $d=\max \left\{K, d^{\prime}\right\}$, where $d^{\prime}=\frac{(\alpha-r)+\sqrt{(\alpha-r)^{2}+4 r K \beta}}{2 \beta}$ $>0$.

\section{Asymptotic behavior of exercise boundary infinitely far from expiry}

Since $s(t)$ is a strictly increasing function of the time-to-maturity for the American volatility power option, the lower boundary for the optimal exercise boundary $s(t)$ for $t>0$ is obtained by $\lim _{t \rightarrow 0^{+}} s(t)=d$ in the MRSRP and the mean-reverting $3 / 2$ volatility process. It would be interesting to explore whether $s(t)$ is bounded or not as $t \rightarrow \infty$. At the same time, the pricing formula for the perpetual American volatility power option is obtained in the MRSRP and the mean-reverting 3/2 volatility process.

Before solving the ordinary differential equation arising from pricing the perpetual American volatility option, we first introduce the confluent hypergeometric functions of which the integral representations are given as

$$
\Phi(a, b, x)=\frac{\Gamma(b)}{\Gamma(b-a) \Gamma(a)} \int_{0}^{1} e^{x t} t^{a-1}(1-t)^{b-a-1} d t .
$$


In the following theorem, we will provide a pricing formula for the perpetual American volatility power option.

Theorem 3.1. Let $(v, s)$ be the value and the early exercise boundary of a perpetual American volatility power option in MRSRP. Assume $\frac{2 \beta m}{\sigma^{2}}>1$ for the Feller condition. If $n>0$, then $(v, s)$ solves the following free boundary problem

$$
\begin{gathered}
\mathcal{L}_{0}^{M} v(x)=0, \quad 0<x<s, \\
v(s)=s^{n}-K, \quad v^{\prime}(s)=n s^{n-1},
\end{gathered}
$$

where $\mathcal{L}_{0}^{M}$ is defined in 1.3 . The solution is obtained in the form

$$
v(x)=C_{1} \Phi\left(\frac{r}{\beta}, \frac{2 \beta m}{\sigma^{2}} ; \frac{2 \beta}{\sigma^{2}} x\right), \quad 0<x<s,
$$

where $C_{1}=\frac{s^{n}-K}{\Phi\left(\frac{r}{\beta}, \frac{2 \beta m}{\sigma^{2}} ; \frac{2 \beta}{\sigma^{2}} s\right)}$ and $s$ is a root of the following equation

$$
\frac{\Phi\left(\frac{r}{\beta}, \frac{2 \beta m}{\sigma^{2}} ; \frac{2 \beta}{\sigma^{2}} s\right)}{\frac{\sigma^{2} r}{2 \beta^{2} m} \Phi\left(\frac{r}{\beta}+1, \frac{2 \beta m}{\sigma^{2}}+1 ; \frac{2 \beta}{\sigma^{2}} s\right)}=\frac{s^{n}-K}{n s^{n-1}},
$$

which can be solved iteratively for $s$.

If $n<0$, then $(v, s)$ solves the following free boundary problem

$$
\begin{gathered}
\mathcal{L}_{0}^{M} v(x)=0, \quad s<x<\infty, \\
v(s)=s^{n}-K, \quad v^{\prime}(s)=n s^{n-1}, \quad \lim _{x \rightarrow \infty} v(x)=0,
\end{gathered}
$$

where $\mathcal{L}_{0}^{M}$ is defined in 1.3 . The solution is obtained in the form

$$
v(x)=\left(s^{n}-K\right)\left(\frac{x}{s}\right)^{1-\frac{2 \beta m}{\sigma^{2}}} \frac{\Phi\left(\frac{r}{\beta}+1-\frac{2 \beta m}{\sigma^{2}}, 2-\frac{2 \beta m}{\sigma^{2}} ; \frac{2 \beta}{\sigma^{2}} x\right)}{\Phi\left(\frac{r}{\beta}+1-\frac{2 \beta m}{\sigma^{2}}, 2-\frac{2 \beta m}{\sigma^{2}} ; \frac{2 \beta}{\sigma^{2}} s\right)}, \quad s<x<\infty .
$$

Here $s$ is a root of the following equation

$$
\frac{s^{1-\frac{2 \beta m}{\sigma^{2}}} \Phi\left(\frac{r}{\beta}+1-\frac{2 \beta m}{\sigma^{2}}, 2-\frac{2 \beta m}{\sigma^{2}} ; \frac{2 \beta}{\sigma^{2}} s\right)}{\left.\frac{d}{d x}\left[x^{1-\frac{2 \beta m}{\sigma^{2}}} \Phi\left(\frac{r}{\beta}+1-\frac{2 \beta m}{\sigma^{2}}, 2-\frac{2 \beta m}{\sigma^{2}} ; \frac{2 \beta}{\sigma^{2}} x\right)\right]\right|_{x=s}}=\frac{s^{n}-K}{n s^{n-1}},
$$

which can be solved iteratively for $s$.

Proof. By letting $y=\frac{2 \beta}{\sigma^{2}} x, \mathcal{L}_{0}^{M} v(x)=0$ is changed to

$$
y \frac{d^{2} v}{d y^{2}}+\left(\frac{2 \beta m}{\sigma^{2}}-y\right) \frac{d v}{d y}-\frac{r}{\beta} v=0, \quad 0<y<\frac{2 \beta}{\sigma^{2}} s,
$$

which is regarded as a Kummer's equation. 
The solutions of Kummer's equation (3.4) are expressed through the confluent hypergeometric function $\Phi(\alpha, \nu, x)$. Precisely, the general solutions are written in the form

$$
v(y)=C_{1} \Phi\left(\frac{r}{\beta}, \frac{2 \beta m}{\sigma^{2}} ; y\right)+C_{2} y^{1-\frac{2 \beta m}{\sigma^{2}}} \Phi\left(\frac{r}{\beta}+1-\frac{2 \beta m}{\sigma^{2}}, 2-\frac{2 \beta m}{\sigma^{2}} ; y\right),
$$

where $C_{1}$ and $C_{2}$ are arbitrary constants.

Displaying the solution in terms of $x$, the equation is rewritten in the form

$$
v(x)=C_{1} \Phi\left(\frac{r}{\beta}, \frac{2 \beta m}{\sigma^{2}} ; \frac{2 \beta}{\sigma^{2}} x\right)+C_{2}\left(\frac{2 \beta}{\sigma^{2}} x\right)^{1-\frac{2 \beta m}{\sigma^{2}}} \Phi\left(\frac{r}{\beta}+1-\frac{2 \beta m}{\sigma^{2}}, 2-\frac{2 \beta m}{\sigma^{2}} ; \frac{2 \beta}{\sigma^{2}} x\right) .
$$

We first consider the case of $n>0$. The value $v(x)$ of an American volatility power option is finite in $[0, s]$, that is, $v(s)<\infty$ for all $x \in[0, s]$. Since $\beta m>\frac{1}{2} \sigma^{2}$ and $\Phi(\alpha, \beta ; x) \neq 0$ as $x \rightarrow 0$, we have $\left(\frac{2 \beta}{\sigma^{2}} x\right)^{1-\frac{2 \beta m}{\sigma^{2}}} \rightarrow \infty$ as $x \rightarrow 0$ and $C_{2}=0$. Consequently, the value of the perpetual American volatility option equals to

$$
v(x)=C_{1} \Phi\left(\frac{r}{\beta}, \frac{2 \beta m}{\sigma^{2}} ; \frac{2 \beta}{\sigma^{2}} x\right) .
$$

To determine the free boundary $s$ and the coefficient $C_{2}$, we substitute $v(s)=s^{n}-K$ and $v^{\prime}(s)=n s^{n-1}$ into 3.5 and obtain that

$$
C_{1} \Phi\left(\frac{r}{\beta}, \frac{2 \beta m}{\sigma^{2}} ; \frac{2 \beta}{\sigma^{2}} s\right)=s^{n}-K
$$

and

$$
\left.C_{1} \frac{d}{d x}\left[\Phi\left(\frac{r}{\beta}, \frac{2 \beta m}{\sigma^{2}} ; \frac{2 \beta}{\sigma^{2}} x\right)\right]\right|_{x=s}=n s^{n-1} .
$$

Moreover, we have $\left.\frac{d}{d x}\left[\Phi\left(\frac{r}{\beta}, \frac{2 \beta m}{\sigma^{2}} ; \frac{2 \beta}{\sigma^{2}} x\right)\right]\right|_{x=s}=\frac{\sigma^{2} r}{2 \beta^{2} m} \Phi\left(\frac{r}{\beta}+1, \frac{2 \beta m}{\sigma^{2}}+1 ; \frac{2 \beta}{\sigma^{2}} s\right)$.

Hence, we find that the free boundary satisfies the following nonlinear algebraic equation

$$
\frac{\Phi\left(\frac{r}{\beta}, \frac{2 \beta m}{\sigma^{2}} ; \frac{2 \beta}{\sigma^{2}} s\right)}{\frac{\sigma^{2} r}{2 \beta^{2} m} \Phi\left(\frac{r}{\beta}+1, \frac{2 \beta m}{\sigma^{2}}+1 ; \frac{2 \beta}{\sigma^{2}} s\right)}=\frac{s^{n}-K}{n s^{n-1}} .
$$

When the free boundary $s$ is obtained by the solving the above equation numerically, the coefficient $C_{1}$ is expressed as

$$
C_{1}=\frac{s^{n}-K}{\Phi\left(\frac{r}{\beta}, \frac{2 \beta m}{\sigma^{2}} ; \frac{2 \beta}{\sigma^{2}} s\right)}
$$

We then consider the case of $n<0$. Since $\lim _{x \rightarrow \infty} v(x)=0$, we have $\frac{2 \beta}{\sigma^{2}} x^{1-\frac{2 \beta m}{\sigma^{2}}} \rightarrow 0$ as $x \rightarrow \infty$ and

$$
C_{1} \Phi\left(\frac{r}{\beta}, \frac{2 \beta m}{\sigma^{2}} ; 0\right) \rightarrow 0 \quad \text { as } x \rightarrow \infty
$$


which means $C_{1}=0$. Consequently, we have

$$
v(x)=C_{2}\left(\frac{2 \beta}{\sigma^{2}} x\right)^{1-\frac{2 \beta m}{\sigma^{2}}} \Phi\left(\frac{r}{\beta}+1-\frac{2 \beta m}{\sigma^{2}}, 2-\frac{2 \beta m}{\sigma^{2}} ; \frac{2 \beta}{\sigma^{2}} x\right) .
$$

To determine the free boundary $s$ and the coefficient $C_{2}$, we substitute $v(s)=s^{n}-K$ and $v^{\prime}(s)=n s^{n-1}$ into $(3.6)$ and obtain that

$$
C_{2}\left(\frac{2 \beta}{\sigma^{2}} s\right)^{1-\frac{2 \beta m}{\sigma^{2}}} \Phi\left(\frac{r}{\beta}+1-\frac{2 \beta m}{\sigma^{2}}, 2-\frac{2 \beta m}{\sigma^{2}} ; \frac{2 \beta}{\sigma^{2}} s\right)=s^{n}-K
$$

and

$$
\left.C_{2} \frac{d}{d x}\left[\left(\frac{2 \beta}{\sigma^{2}} x\right)^{1-\frac{2 \beta m}{\sigma^{2}}} \Phi\left(\frac{r}{\beta}+1-\frac{2 \beta m}{\sigma^{2}}, 2-\frac{2 \beta m}{\sigma^{2}} ; \frac{2 \beta}{\sigma^{2}} x\right)\right]\right|_{x=s}=n s^{n-1} .
$$

Hence, we find that the free boundary satisfies the following nonlinear algebra equation

$$
\frac{\left(\frac{2 \beta}{\sigma^{2}} s\right)^{1-\frac{2 \beta m}{\sigma^{2}}} \Phi\left(\frac{r}{\beta}+1-\frac{2 \beta m}{\sigma^{2}}, 2-\frac{2 \beta m}{\sigma^{2}} ; \frac{2 \beta}{\sigma^{2}} s\right)}{\left.\frac{d}{d x}\left[\left(\frac{2 \beta}{\sigma^{2}} x\right)^{1-\frac{2 \beta m}{\sigma^{2}}} \Phi\left(\frac{r}{\beta}+1-\frac{2 \beta m}{\sigma^{2}}, 2-\frac{2 \beta m}{\sigma^{2}} ; \frac{2 \beta}{\sigma^{2}} x\right)\right]\right|_{x=s}}=\frac{s^{n}-K}{n s^{n-1}} .
$$

When the free boundary $s$ is obtained by the solving the above equation numerically, the coefficient $C_{2}$ is expressed as

$$
C_{2}=\frac{s^{n}-K}{\left(\frac{2 \beta}{\sigma^{2}} s\right)^{1-\beta m} \Phi\left(\frac{r}{\beta}+1-\frac{2 \beta m}{\sigma^{2}}, 2-\frac{2 \beta m}{\sigma^{2}} ; \frac{2 \beta}{\sigma^{2}} s\right)} .
$$

Remark 3.2. When $(v, s)$ are the value and the early exercise boundary of a perpetual American volatility option in mean reverting $3 / 2$ volatility process, $(v, s)$ is the solution of the following free boundary problem

$$
\begin{gathered}
\mathcal{L}_{0}^{Q} v(x)=0, \quad 0<x<s \\
v(s)=s-K, \quad v^{\prime}(s)=n s^{n-1}, \quad v(0)=0,
\end{gathered}
$$

where $\mathcal{L}_{0}^{Q}$ is defined in $(1.4)$. When taking $x=1 / y$, this problem becomes to price a perpetual American volatility option in the MRSRP with $\psi(x)=(1 / x-K, 0)^{+}$. Hence, the price of a perpetual American option becomes to satisfy the following free boundary problem

$$
\begin{gathered}
\mathcal{L}_{0}^{M} u(y)=0, \quad 1 / s<y<\infty, \\
u(s)=1 / s-K, \quad u^{\prime}(s)=-1 / s^{2}, \quad u(y) \rightarrow 0 \text { as } y \rightarrow \infty .
\end{gathered}
$$

The solution of this equation can be obtained by changing it to a Kummer's equation. In the mean-reverting $3 / 2$ volatility process, a closed pricing formula for the perpetual American volatility put option is proposed by Liu [10]. 
This paper provides a formula for the perpetual American volatility power call in the MRSRP model. Liu [10] provided a formula for the perpetual American volatility put in the mean-reverting $3 / 2$ volatility model. These two papers considered distinct options (call and put) in the different processes (the MRSRP and the mean-reverting $3 / 2$ volatility process). Using the change of variables, the differential equations in both two papers are changed to the same Kummer's differential equation with the different boundary conditions. The general solution is expresses by the combination of the confluent hypergeometric function of the first and second kinds.

For the volatility call, we have a finite initial condition and an upper free boundary; For the volatility put, we have a lower free boundary and assume that the put value tends to zero as the volatility tends to infinite. The different conditions for both put and call induces different pricing formulas. For the volatility call, we eliminate the second independent solution by the finite initial condition. For the volatility put, we eliminate the first independent solution since the put value tends to zero as the volatility tends to infinite. Moreover, the advantage of this paper is that we add a power to the payoff function, $\psi(x)=\max \left\{x^{n}-K, 0\right\}$. When setting the volatility $x$ to $1 / y$ (i.e., choosing $n=-1$ ), the MRSRP model can be changed to the mean-reverting $3 / 2$ volatility model. In this case, the boundary conditions are changed to a lower free boundary and zero value at the infinite.

\section{Artificial neural network approach}

In this section, we consider neural network (NN) approach to the solution of the free boundary differential equation arising from pricing a perpetual American volatility option under the MRSRP.

Alternatively, this NN approach can also be applied to approximate the pricing formula of the perpetual American option under the different process.

We first state a formal definition of a neural network used in this paper.

Definition 4.1. A neural network (NN) is a function $f_{\Theta}: \mathbb{R}^{n} \rightarrow \mathbb{R}^{m}$ defined by a series of alternative compositions of affine and non-linear functions with a set of parameters $\Theta=\left\{\left(A_{i}, b_{i}\right): 0 \leq i \leq n\right\}$ of the following form

$$
f=L_{n} \circ \sigma_{n-1} \circ L_{n-1} \circ \cdots \sigma_{0} \circ L_{0}
$$

where $L_{i}: \mathbb{R}^{h_{i}} \rightarrow \mathbb{R}^{h_{i+1}}$ is an affine transformation defined by $L_{i}(x)=A_{i} x+b_{i}, \sigma_{i}: \mathbb{R}^{h_{i+1}} \rightarrow$ $\mathbb{R}^{h_{i+1}}$ is a non-linear activation function. Note that activation function operates on vectors component-wisely. That is, $\sigma(x)=\left(\sigma\left(x_{1}\right), \sigma\left(x_{2}\right), \ldots, \sigma\left(x_{n}\right)\right)$ for $x=\left(x_{1}, \ldots, x_{n}\right) \in \mathbb{R}^{n} . n$ is called the depth of the $\mathrm{NN}$ and $h_{i}$ is the width of the $i$-th layer of the NN, for $i \geq 1$. 
Note that an NN is called a shallow net if $n=1$, and it is called a deep neural network for $n \geq 3$.

Hornik, Stinchcombe and White [7] show that any Borel measure function could be approximated in any degree of accuracy by a shallow net, provided a sufficient number of neurons in the hidden layer. In this paper, we consider a special activation function, called Swish function defined by $\frac{x}{1+e^{-x}}$. For more detail of this activation function, please refer to 12 .

Now, we go back to the NN approach to our MRSRP problem. Suppose that $\frac{2 \beta m}{\sigma^{2}}>1$ and $(v, s)$ are the value and the early exercise boundary of a perpetual American volatility power option in MRSRP. Then $(v, s)$ is the solution the free boundary problem 3.3. Transferring $y=\frac{x}{s}$ and defining $w(y)=v(x)$, the free boundary problem is changed to the following boundary value problem

$$
\frac{1}{2} \sigma^{2} y w^{\prime \prime}+\beta(m-s y) w^{\prime}-r s w=0, \quad 0<y<1
$$

with $w(1)=s^{n}-K$ and $w^{\prime}(1)=n s^{n-1}$. Substituting $s=(w(1)-K)^{1 / n}$ into 4.1 yields

$$
\frac{1}{2} \sigma^{2} y w^{\prime \prime}+\beta\left(m-(w(1)+K)^{1 / n} y\right) w^{\prime}-r(w(1)+K)^{1 / n} w=0, \quad 0<y<1
$$

with $w^{\prime}(1)=n(w(1)+K)^{(n-1) / n}$. We consider the following trial solution

$$
w_{A}(y)=f_{\Theta}(y)
$$

where $f_{\Theta}: \mathbb{R}^{2} \rightarrow \mathbb{R}$ is a shallow net defined by $\Theta$. Then, we obtain $s=\left(f_{\Theta}(y)+K\right)^{1 / n}$ and $w_{A}^{\prime}(1)=n\left(f_{\Theta}(1)+K\right)^{(n-1) / n}$. Therefore, we turn into solve the following equation

$$
\frac{1}{2} \sigma^{2} y w_{A}^{\prime \prime}(y)+\beta\left(m-\left(f_{\Theta}(1)+1+K\right)^{1 / n} y\right) w_{A}^{\prime}(y)-r\left(f_{\Theta}(1)+1+K\right)^{1 / n} w_{A}(y)=0
$$

with $w_{A}^{\prime}(1)=n\left(f_{\Theta}(1)+K\right)^{(n-1) / n}$.

To find an optimal $f_{\Theta}$ to solve the above equations, we consider the following minimization problem

$$
\min _{\Theta} L(\Theta)
$$

where

$$
L(\Theta)=\int_{0}^{1}\left[\frac{1}{2} \sigma^{2} y w_{A}^{\prime \prime}(y)+\beta(m-s y) w_{A}^{\prime}(y)-r s w_{A}(y)\right]^{2}+\left[w_{A}^{\prime}(1)-n s^{n-1}\right]^{2} d y
$$

is the $L^{2}$-loss between $\mathrm{NN}$ and the solution of equation and the boundary condition, and $s=\left(f_{\Theta}(1)+K\right)^{1 / n}$. 
In addition, an NN could be write down as follows:

$$
f_{\Theta}(y)=\sum_{k=1}^{H_{M}} \omega_{k, i}^{M} \sigma\left(S_{M, k}\right)
$$

Here, the output neuron $i$ in layer $j s_{j, i}$ is defined by

$$
s_{1, i}=w_{y, i}^{0} y+w_{b, i}^{1}, \quad s_{j, i}=\sum_{k=1}^{H_{j-1}} \omega_{k, i}^{j-1} \sigma\left(s_{j-1, k}\right)+\omega_{b, i}^{j}, \quad j=1,2, \ldots, M,
$$

where $\omega_{k, i}^{j}$ is the weight from neuron $k$ in layer $j-1$ to neuron $i$ in layer $j$ for the network $f_{\Theta}(y)$, and $H_{j}$ is the number of neurons in layer $j$ and $\omega_{b, i}^{j}$ is the bias of neuron $i$ in layer $j$.

5. Comparison results between the analytical solution and the numerical solution

In Section 3, we provided an analytical pricing formula (3.1) for the perpetual American power call option by solving the Kummers equation (3.4), where the early exercise boundary $s$ is obtained by solving (3.2) numerically. In Section 4, we developed a new numerical method to approximate the differential equation with moving boundary by extending the artificial neural network approach. In this section, we will demonstrate the comparison results between the analytical solution and the numerical solution.

Goard and Mazur [5] used the data of the VIX index value between years of 1990 and 2009 to estimate the parameters the continuous-time model. In the empirical results, the parameters are estimated as $\beta=3.1637$ and $\beta m=0.6154$ (see [5. Table 5.1]) for the MRSRP model. Moreover, the risk-free interest rate and strike are given as $r=0.05$ and $K=0.5$, respectively. To satisfy $\frac{2 \beta m}{\sigma^{2}}>1$, we assume $\sigma^{2}=1$.

To compare the analytical solution and the numerical solution, the graphs for the price of perpetual American power call options are demonstrated. The programs are coded by Python 11 on Google Colab environment. The solution of the early exercise boundary $s$ is solved by using the "fsolve" instruction, where the starting estimate for the roots is given as $K+1$. The network is constructed by 1 input layer, 1 output layer and 1 hidden layer with 10 neurons in the hidden layer. For the neural network approach, the deep learning algorithm, Adam [8], is used to minimize the unconstrained optimization problem.

The $L^{2}$-losses between NN and the solution of equation and the boundary condition are $2.31 e-4,5.19 e-5$ and $2.34 e-5$ for 10000 iterations, 20000 iterations and 30000 iterations. The $L^{2}$-losses for 20000 iterations and 30000 iterations are same as $e-5$ and do not reduce so much from 20000 iterations to 30000 iterations. The comparison results between 
the analytical solution and the numerical soltion (20000 iterations) are demonstrated in Figure 5.1 and Figure 5.2 for 10000 iterations and 20000 iterations, respectively.

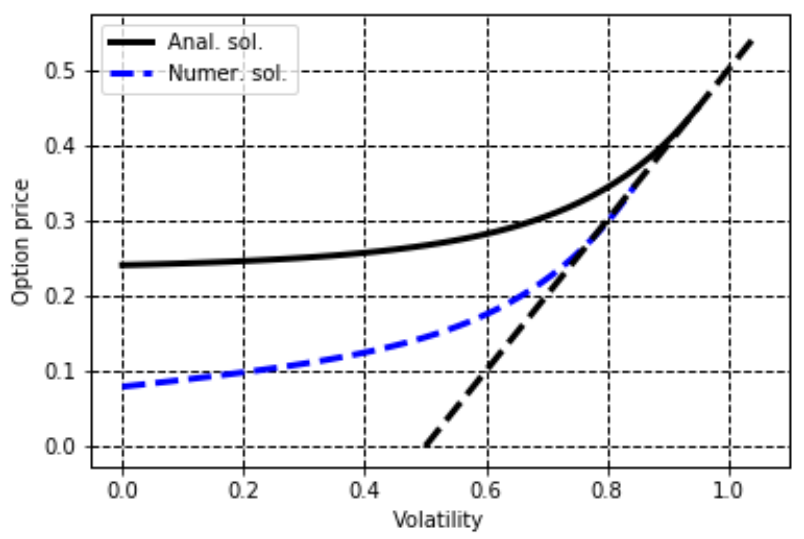

Figure 5.1: The comparison results between analytic solution and the numerical solution (10000 iterations).

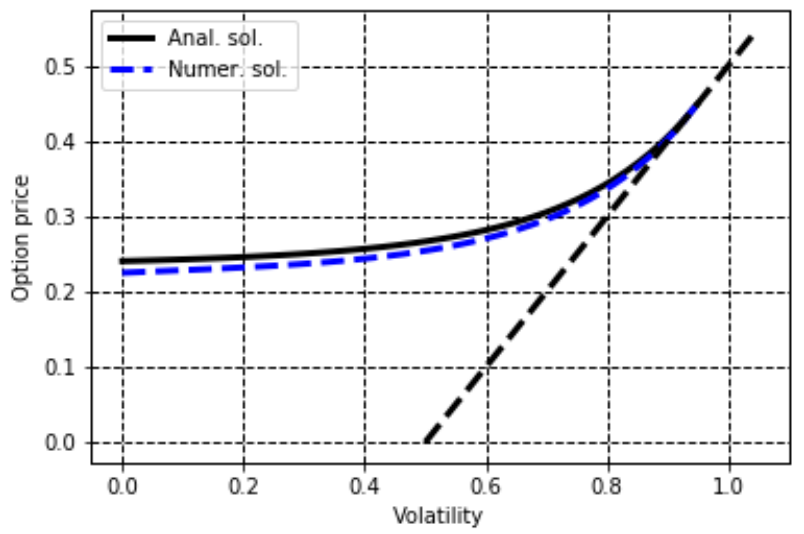

Figure 5.2: The comparison results between analytic solution and the numerical solution (20000 iterations).

\section{References}

[1] J. C. Cox, J. E. Ingersoll and S. A. Ross, A theory of the term structure of interest rates, Econometrica 53 (1985), no. 2, 385-407.

[2] J. Detemple and Y. Kitapbayev, On American VIX options under the generalized $3 / 2$ and 1/2 models, Math. Finance 28 (2018), no. 2, 550-581. 
[3] J. Detemple and C. Osakwe, The valuation of volatility options, Rev. Finance 4 (2000), no. 1, 21-50.

[4] W. Feller, Two singular diffusion problems, Ann. of Math. (2) 54 (1951), 173-182.

[5] J. Goard and M. Mazur, Stochastic volatility models and the pricing of VIX options, Math. Finance 23 (2013), no. 3, 439-458.

[6] A. Grünbichler and F. A. Longstaff, Valuing futures and options on volatility, J. Bank. Finance 20 (1996), no. 6, 985-1001.

[7] K. Hornik, M. Stinchcombe and H. White, Multilayer feedforward networks are universal approximators, Neural Netw. 2 (1989), no. 5, 359-366.

[8] D. P. Kingma and J. Ba, Adam: A method for stochastic optimization, available at arXiv:1412.6980.

[9] D. B. Kotlow, A free boundary problem connected with the optimal stopping problem for diffusion processes, Trans. Amer. Math. Soc. 184 (1973), 457-478.

[10] H.-K. Liu, Properties of American volatility options in the mean-reverting $3 / 2$ volatility model, SIAM J. Finan. Math. 6 (2015), no. 1, 53-65.

[11] Python Software Foundation, Python Language Reference, version 2.7. Available at https://www.python.org.

[12] P. Ramachandran, B. Zoph, Q. V. Le, Searching for activation functions, available at arXiv:1710.05941.

\section{Hsuan-Ku Liu}

Department of Mathematics and Information Education, National Taipei University of Education, Taiwan

E-mail address: hkliu.nccu@gmail.com

Tse-Yu Lin and Yen-Lung Tsai

Department of Mathematical Sciences, National Chengchi University, Taiwan

E-mail addresses: orochi77912@gmail.com, yenlung@nccu.edu.tw 